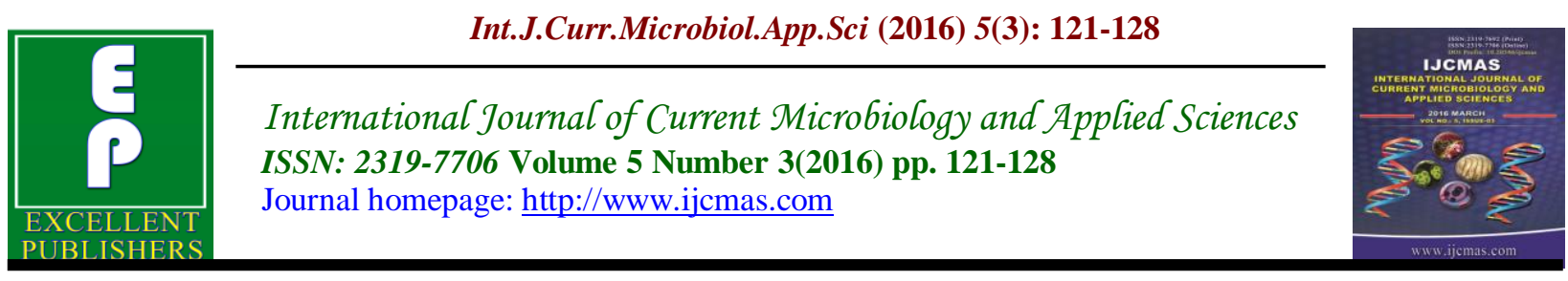

Original Research Article

http://dx.doi.org/10.20546/ijcmas.2016.503.017

\title{
Resistance of Antimicrobial in Pseudomonas aeruginosa
}

\section{Introduction}

Antimicrobial agents have been the only easily and widely used therapeutic option available to counter the infections caused by infectious microbial agents. However, microbial populations have developed various strategies to overcome these microbial agents - a major contributing factor in the development anti-microbial resistance world-wide.
Pseudomonas aeruginosa is an aerobic, motile, nutritionally versatile, gram negative bacteria. Pseudomonas aeruginosa is ubiquitous, human opportunistic pathogen and has implications on morbidity, mortality and healthcare costs both in hospitals and in the community (Franco BE et al., 2009). Infections caused by Pseudomonas aeruginosa is frequently life threatening and 
difficult to treat as it exhibits intrinsically high resistance to many antimicrobials and the development of increased, particularly multi drug resistance in health care settings (Poole K 2011). Ongoing surveillance of Pseudomonas aeruginosa resistance against antimicrobial agents is fundamental to monitor trends in susceptibility pattern and to appropriately guide the clinicians in choosing empirical or directed therapy, especially when new antimicrobial agents may not be readily available in the near future (Gales AC et al., 2001). Ongoing studies on current antimicrobial resistance profile of $\mathrm{P}$. aeruginos are essential to find out the susceptibilities of this pathogen against commonly prescribed antibiotics in any health care facility. This would help the physicians to optimize the current therapeutics treatment options. Thus, in our study we assessed the in vitro activity level of antimicrobial drugs against clinical isolates of Pseudomonas aeruginosa obtained from the NIMS Medical College \& Hospital, Jaipur.

\section{Materials and Methods}

This investigation was carried out in the Department of Microbiology, NIMS Medical College \& Hospital, Jaipur, Rajasthan, India during August 2015 to December 2015. Specimens were collected from patients who were hospitalized for more than one week duration. A total 126 consecutive clinical isolates of $P$. aeruginosa were collected for bacterial culture and identification. Only one isolate from each patient was considered in this study.

\section{Sample Processing}

The specimens were collected from the hospitalized patients admitted from different wards of hospital. These were processed for bacterial species identification by standard microbiological procedures. Specimens were taken from various sources like pus/wound, sputum, urine, bronchoalveolar lavage (BAL) fluid, tracheal aspirate and were inoculated on routine culture media like Blood agar, MacConkey agar. MacConkey agar showed lactose nonfermenting pale colonies with oxidase positive.

\section{Conformation of Pseudomonas spp}

After obtaining the pure strains, the strains subjected the grams staining and biochemical identification tests to identify Pseudomonas spp. For this purpose the samples are inoculated with Peptone water, Urease media, Citrate, TSI (Triple Sugar Iron) media and kept an incubator at $37^{\circ} \mathrm{C}$ for $18 \mathrm{hrs}$. Next day the result will noted on Citrate media, Urease media, TSI media. Part of growth on peptone water was subjected to indole test with Kovac, s reagent and part for motility testing by Hanging drop method. A strain of Pseudomonas showed Indole negative, Urease test negative, TSI medium showed alkaline slant and no reaction in butt and Citrate test positive. Nitrate reduction test was positive in Pseudomonas (Konemen, 2006).

\section{Antimicrobial Susceptibility Testing}

Antimicrobial susceptibility testing of all the Pseudomonas aeruginosa isolates was performed by Kirby-Bauer disk diffusion method and the result were interpreted by the Clinical Laboratory Standard Institute (CLSI) guidelines 2014. All the clinical isolates of $P$. aeruginosa were tested for their sensitivity against a panel of antipseudomonal antimicrobials of standard strength as follow: Amikacin $30 \mathrm{mcg}$, Piperacillin $100 \mathrm{mcg}$, Ceftriaxone $30 \mathrm{mcg}$, 
Cefoperazone-Sulbactum $\quad 75-10 \quad \mathrm{mcg}$, Ciprofloxacin 5mcg, Co-Trimoxazole $25 \mathrm{mcg}$, Imipenem $10 \mathrm{mcg}$ and Meropenem 10mcg (Hi Media Laboratories Pvt. Ltd., Mumbai, India). $P$. aeruginosa ATCC 27853 was used as quality control strain.

\section{Results and Discussion}

A total 126 strains of Pseudomonas aeruginosa were isolated and identified by standard microbiological procedures, out of a total 654 clinical specimens were investigated. The rate of isolation of $P$. aeruginosa was 126 (19.26\%). Of these 126 strains of P. aeruginosa, $78(61.90 \%)$ were from males and 48(38.09\%) from females patients shown in Table 1 . Most of them belonging from the age group 21-40 $(45.23 \%)$ years followed by patients of $>60$ years $(24.60 \%)$ of age as shown in Table 2. Wound/Pus, Sputum, Urine and Tracheal Aspirate were the predominant source of specimens of $P$. aeruginosa clinical isolates as shown in Table 3.

In our study, a total of 126 isolates of Pseudomonas aeruginosa were isolated and identified from various clinical specimens from the hospitalized patients and their antimicrobial sensitivity determined. Most of them are belong to older age group of 2140 years $(45.23 \%)$ and elderly age group $>60$ years $(24.60 \%)$. This could be explained as due to decreased immunity, prolonged hospitalization and other associated co-morbidities in these age groups.

A study done in Ahmadabad in Gujarat state of India shown (29\%) of patients were aged between 31-45 years (Rajat RM et al., 2012). Similarly, a high prevalence of $P$. aeruginosa infection was found in the 35-50 years age group (Mohanasoundaram KM 2011). The distribution of specimens of $P$. aeruginosa may vary with each hospital as each hospital facility has a different environment associated with it. More than $80 \%$ of the $P$. aeruginosa isolates were obtained from Pus/Wound, Urine and Tracheal Aspirates. Increasing resistance to different anti-pseudomonal drugs particularly among hospital strains has been reported world-wide (Orrett FA. 2004) and this is a serious therapeutic problem in the management of disease due to these organisms. The resistance profile of Pseudomonas aeruginosa to the eight antimicrobial agents tested varied among the isolate investigated. One streaking feature in our study was that all the $P$. aeruginosa isolates were found to be sensitive to Imipenem and Meropenem. This may due to restricted use of Imipenem and Meropenem in our hospital. Amikacin (18.2\%) and Ciprofloxacin (31.7\%) show very low resistance and proved to be the most effective drugs for routine use among the $P$. aeruginosa strains investigated in this study. An earlier study reported from Nepal, shown Amikacin (81.4\%) and Ciprofloxacin (70.3\%) are high sensitive drugs against $P$. aeruginosa (Koirala $\mathrm{P}$ et al., 2010).

Murase, et al. 1995 in their study showed that there is distinct difference in the sensitivity pattern of isolates of $P$. aeruginosa from specimen to specimen. Piperacillin alone tested showed a resistance rate of $(53.9 \%)$ in this study wears beta lactams/ beta-lactams inhibitor drug Cefoperazone-Sulbactum showed a lower resistance of $(36.5 \%)$ only. The emphasis should be given towards use of combined antibiotics in the treatment of Pseudomonal infections (Bhandari S et al., 2012). Similar resistance rate for Piperacillin (54.6\%) has been reported in the study done by Shenoy et al. 2002. Relatively low Piperacillin resistance $(11.5 \%)$ had been reported in patient isolates of $P$. aeruginosa in a study from Saudi Arabia (Al -Tawfiq JA. 2007). 
Table.1 Sex Wise Distribution of Cases

\begin{tabular}{|c|c|c|}
\hline Sex & Total no. & Percentage (\%) \\
\hline Male & 78 & $61.90 \%$ \\
\hline Female & 48 & $38.09 \%$ \\
\hline Total & 126 & $100 \%$ \\
\hline
\end{tabular}

Table.2 Age Distribution of Cases

\begin{tabular}{|c|c|c|}
\hline Age (Years) & No. of Isolates & Percentage (\%) \\
\hline$<\mathbf{2 0}$ & 17 & $13.49 \%$ \\
\hline $\mathbf{2 1}-\mathbf{4 0}$ & 57 & $45.23 \%$ \\
\hline $\mathbf{4 1}-\mathbf{6 0}$ & 21 & $16.66 \%$ \\
\hline$>\mathbf{6 0}$ & 31 & $24.60 \%$ \\
\hline Total & 126 & $100 \%$ \\
\hline
\end{tabular}

Table.3 Distribution of $P$. aeruginosa from Different Clinical Samples

\begin{tabular}{|c|c|c|}
\hline Source of specimen & No. of Specimens & Percentage (\%) \\
\hline Pus & 54 & $42.85 \%$ \\
\hline Sputum & 32 & $25.39 \%$ \\
\hline Urine & 23 & $18.25 \%$ \\
\hline Tracheal Aspirate & 11 & $8.73 \%$ \\
\hline BAL & 06 & $4.76 \%$ \\
\hline Total & 126 & $100 \%$ \\
\hline
\end{tabular}

Table.4 Antimicrobial Resistance Pattern of Pseudomonas aeruginosa Isolated from Different Clinical Samples

\begin{tabular}{|c|c|c|}
\hline Antibiotic & No. of Isolate Resistance & \% Resistance \\
\hline Amikacin & 23 & 18.25 \\
\hline Piperacillin & 68 & 53.96 \\
\hline Ceftriaxone & 94 & 76.60 \\
\hline Cefoperazone- & 46 & 36.50 \\
\hline Sulbactum & 38 & 30.15 \\
\hline Ciprofloxacin & 40 & 31.74 \\
\hline Co-Trimoxazole & 91 & 72.22 \\
\hline Imipenem & 00 & 00 \\
\hline Meropenem & 00 & 00 \\
\hline
\end{tabular}


Int.J.Curr.Microbiol.App.Sci (2016) 5(3): 121-128

Fig. 1: Sex wise distribution of cases

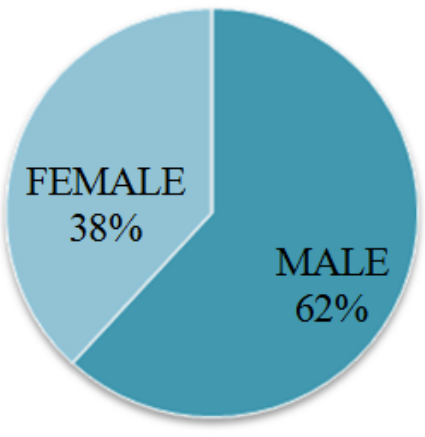

Fig. 2: Age Distribution of cases

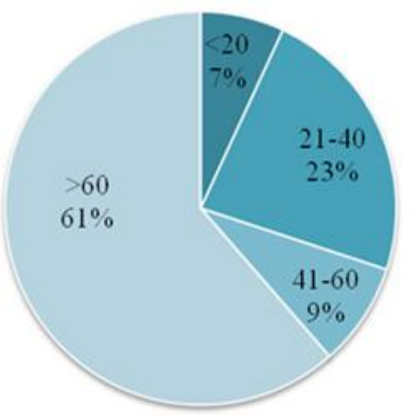

Fig. 3: Distribution of $P$. aeruginosa from different clinical samples

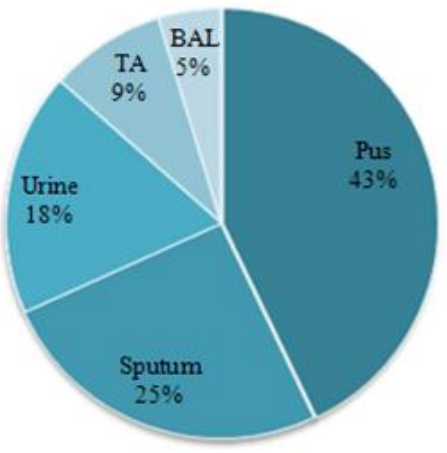


Fig.4 Antimicrobial Resistance Pattern of Pseudomonas aeruginosa Isolated from Different Clinical Samples

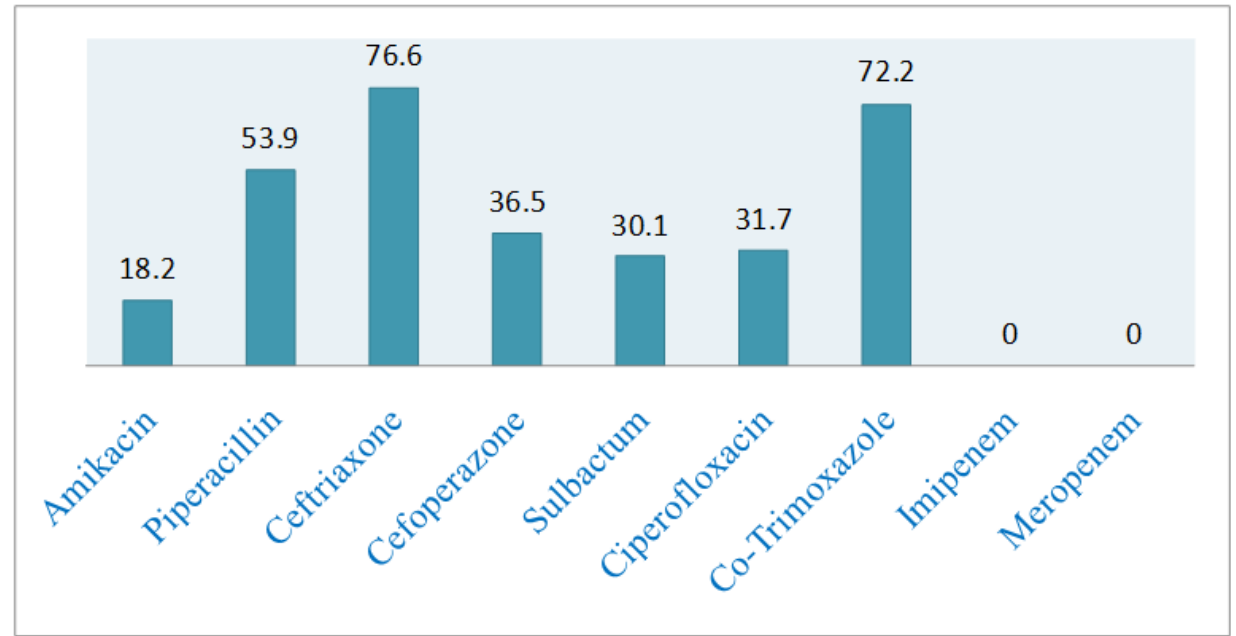

A study of Bhandari S et al., 2012 showed $P$. aeruginosa isolates obtained from intensive care unit (ICU) of National Heart Centre has high Cefoperazone-Sulbactum sensitivity rate of $(84.8 \%)$ and another study of Ahmed SM et al.,. 2012 has been showed low resistance in Cefoperazone-Sulbactum $(11.1 \%)$. The rate of resistance for $\mathrm{Co}$ Trimoxazole on the present study was (72.2\%). In contrast, a study of Rashid A et al., 2007 has been showed rate of resistance for Co-Trimoxazole to be $(93.5 \%)$ in wound swabs and Pus Isolates, while a study of Nwankwo EOK et al.,. 2010 showed $P$. aeruginosa isolates (100\%) resistance to CoTrimoxazole. Pseudomonas aeruginosa strains in this study exhibited a high rate of resistance to the third generation cephalosporin drug- Ceftriaxone (76.6\%). A much high resistance to Ceftriaxone of (75\%) had been reported in study done by Arora D et al., 2011. Lesser rate of resistance to Ceftriaxone $(40 \%)$ had been reported in another study of Ramana BV et al., 2012.

Our study thus indicates that $P$. aeruginosa is becoming resistant to commonly used antibiotics due to excessive consumption of antibiotics exerting selected pressure on bacteria, frequently used invasive devices and severs under laying diseases. The empirical antibiotic treatment should be avoided and treatment should be carried out using antibiotic susceptibility test and efforts should be made to prevent spread of resistant bacteria.

In conclusion, Result of the present study clearly demonstrated the occurrence of resistance to various antipseudomonal agents among the $P$. aeruginosa isolates. Imipenem and Meropenem was the only antipseudomonal drugs against which all isolates of $P$. aeruginosa were fully sensitive. We suggest a more restricted and a more rational use of these drugs in this hospital setting. Amikacin, Ciprofloxacin and semi-synthetic penicillin with beta lactamase inhibitors are the preferred drugs for optimal management of infection caused by Pseudomonas aeruginosa. Regular antimicrobial susceptibility monitoring is essential of local, regional and national level isolates. This would held and guide the physicians in prescribing the right 
combination of anti-microbial to limit and prevent the emergency of multi-drug resistant strains of Pseudomonas aeruginosa.

\section{References}

Ahmed, S.M., Jakribettu, R.P., Kotakutty, S., Arya, B., Shakir, V.P.A. 2012. An emerging multi-drug resistance pathogen in a tertiary care centre in North Kerala. Annal. Biol. Res., 3(6): 2794-2799.

Al-Tawfiq, J.A. 2007. Occurrence and antimicrobial resistance pattern of inpatient and outpatient isolates of $P$. aeruginosa in a Saudi Arabian hospital. Int. J. infect. Dis., 11: 109114.

Arora, D., Jindal, N., Kumar, R., Romit. 2011. Emerging antibiotic resistance in P. aeruginosa. Int. J. Pharm. Sci., 3(2): 82-84.

Bhandari, S., Banjara, M.R., Lekhak, B., Bhatta, D.R., Regmi, S.R. 2012. Multi-drug and pan-drug resistant $P$. aeruginosa a challenge in postantibiotic era. Nepal. J. Sci. Tech., 13(2): 197-202.

CLSI. 2014. Performance standards for antimicrobial susceptibility testing twenty-fourth informational supplement. CLSI document M100s24. Clinical Laboratory Standard Institute, Wayne, PA.

Franco, B.E., Martinez, M.A., Rodriguez, M.A.S., Wertheimer, A.I. 2009. The determinants of the antibiotic resistance process. Infect. Drug. Resist., 2: 1-11.

Gale, A.C., Jones, R.N., Turnidge, J., Rennie, R., Ramphal, R. 1997-1999. Characterization of Pseudomonas aeruginosa isolates: occurrence rate, antimicrobial susceptibility patterns, and molecular typing in the global
SENTRY Antimicrobial Surveillance Program. Clin. Infect. Dis., 32: S146155.

Koneman. 2006. The non-fermentative gram negative bacilli. In .Koneman's color atlas and textbook of diagnostic microbiology, Sixth edn., Williams \& Wikins, Lippincott. pp. 301-391.

Koriala, P., Bhatta, D.R., Ghimire, P., Pokhrel, B.M., Devkota, U. 2010. Bacteriological profile of tracheal aspirate of the patients attending a neuro-hospital of Nepal. Int. J. Life. Sci., 4: 60-65.

Mohanasoundaram, K.M. 2011. The antibiotic resistant pattern in clinical isolates of Pseudomonas aeruginosa in a tertiary care hospital; 20082010. J. Clin. Diagn. Res., 5(3): 491494.

Murase, M., Miyamoto, H., Handa, T., Shaki, S., Takenchi. 1995. Activity of the antipseudomonal agents against clinical isolates of $\mathrm{P}$. aeruginosa. Jpn. J. Antibiot., 48(10): 1581-1589.

Nwankwo, E.O.K., Shuaibo, S.A. 2010. Antibiotics susceptibility pattern of clinical isolates of $P$. aeruginosa in a tertiary health Institute in Kano, Nigeria. J. Med. Biomed. Sci., 37-40.

Orrett, F.A. 2004. Antimicrobial susceptibility survey of Pseudomonas aeruginosa strains isolated from clinical sources. J. Natl. Med. Assoc., 96(8): 1065-1069.

Poole, K. 2011. Pseudomonas aeruginosa: resistance to the max. Front Microbiol., 2: 1-13.

Rajat, R.M., Ninama, G.L., Mistry, K., Parmar, R., Patel, K., Vegad, M.M. 2012. Antibiotic resistance pattern in Pseudomonas aeruginosa species isolated at a tertiary care hospital, Ahmadabad. Nat. J. Med. Res., 2(2): 156-159. 
Ramona, B.V., Chaudhury, A. 2012. Antibiotic resistance pattern of Pseudomonas aeruginosa isolated from health care associated infections at a tertiary care hospital. J. Sci. Sco., 39: 78-80.

Rashid, A., Chowdhury, A., Rahman, S.H.Z., Begum, S.A., Muazzam, N. 2007. Infection by $P$. aeruginosa and antibiotic resistance pattern of isolates from Dhaka Medical College Hospital, Bangladesh. J. Med. Microbiol., 1(2): 48-51.

Shenoy, S., Baliga, S., Saldhanha, D.R., Prashanth, H.V. 2002. Antibiotic sensitivity patterns of $P$. aeruginosa isolated from various clinical specimens. Ind. J. Med. Sci., 56(9): 427-430.

\section{How to cite this article:}

Sheetal Sharma and Preeti Srivastava. 2016. Resistance of Antimicrobial in Pseudomonas aeruginos. Int.J.Curr.Microbiol.App.Sci. 5(3): 121-128.

doi: http://dx.doi.org/10.20546/ijcmas.2016.503.017 